\title{
Tetraspanin 7 autoantibodies in type 1 diabetes
}

\author{
Denise Walther $^{1}$ - Anne Eugster ${ }^{1} \cdot$ Sibille Jergens $^{2}$ • Anita Gavrisan ${ }^{3}$. \\ Christina Weinzierl $^{4}$ - Tanja Telieps ${ }^{4} \cdot$ Christiane Winkler $^{2}$. \\ Anette G. Ziegler ${ }^{2,3}$ • Ezio Bonifacio ${ }^{1,5}$
}

Received: 14 March 2016 / Accepted: 4 May 2016 /Published online: 25 May 2016

(C) Springer-Verlag Berlin Heidelberg 2016

\begin{abstract}
Aims/hypothesis Autoantibodies to pancreatic beta cell proteins are markers of asymptomatic type 1 diabetes. The aim was to determine whether autoantibodies to the beta cell protein tetraspanin 7 would improve the ability to identify autoimmunity against pancreatic beta cells.

Methods Full length and external domain fragments of tetraspanin 7 were expressed as luciferase-tagged fusion proteins and used in immunoprecipitation assays to measure autoantibodies in samples from 363 patients with type 1 diabetes at onset of disease, 503 beta cell autoantibody negative firstdegree relatives of patients, and 212 relatives with autoantibodies to insulin, glutamic acid decarboxylase, insulinoma antigen 2 or zinc transporter 8 .

Results Antibody binding was observed against the full length and external domains of tetraspanin 7, and was strongest against the full length protein. Autoantibodies that could be inhibited by untagged tetraspanin 7 were detected in $5(1 \%)$ of 503 autoantibody negative relatives, 3 (3.2\%) of 94
\end{abstract}

Ezio Bonifacio

ezio.bonifacio@crt-dresden.de

1 DFG Research Center for Regenerative Therapies Dresden, Faculty of Medicine, Technische Universität Dresden, Fetscherstrasse 105, 01307 Dresden, Germany

2 Institute of Diabetes Research, Helmholtz Zentrum München, and Forschergruppe Diabetes, Klinikum rechts der Isar, Technische Universität München, Neuherberg, Germany

3 Forschergruppe Diabetes e.V., Neuherberg, Germany

4 Institute of Diabetes and Obesity, Helmholtz Zentrum München, Neuherberg, Germany

5 Paul Langerhans Institute Dresden, German Center for Diabetes Research (DZD), Technische Universität Dresden, Dresden, Germany autoantibody negative patients, 95 (35.3\%) of 269 autoantibody positive patients, 1 (1\%) of 98 single autoantibody positive relatives and 25 (21.9\%) of 114 multiple autoantibody positive relatives. Progression to diabetes did not differ between multiple autoantibody positive relatives with and without tetraspanin 7 autoantibodies.

Conclusions/interpretation Tetraspanin 7 is an autoantigen in type 1 diabetes. Tetraspanin 7 autoantibodies are a marker of type 1 diabetes, but provide minor additional value to existing autoantibodies in identifying beta cell autoimmunity.

Keywords Autoantibodies · Glima $38 \cdot$ Tetraspanin $7 \cdot$ Type 1 diabetes mellitus
Abbreviations
ED Extracellular domain
IA-2 Insulinoma antigen-2
ZnT8 Zinc transporter 8

\section{Introduction}

Glima 38 is an N-glycosylated amphiphilic transmembrane protein of the pancreatic beta cell $[1,2]$. Two reports estimate the prevalence of glima 38 autoantibodies, measured by complex immuno-precipitation assays of radio-labelled cell lines, to be around 20 to $40 \%$ in patients with type 1 diabetes [1,3]. The recent identification of glima 38 as tetraspanin 7 [4] allows the development of less cumbersome recombinant protein-based immunoassays for the measurement of these autoantibodies. Here, we developed a luciferase reporter immunoassay for tetraspanin 7 autoantibodies, and have quantified tetraspanin 7 autoantibodies in individuals with increased genetic risk for type 1 diabetes and at onset of type 1 diabetes 
in order to determine whether tetraspanin 7 autoantibodies improve our ability to identify autoimmunity against the pancreatic beta cell.

\section{Methods}

Participants Sera were selected from the DiMelli cohort of new onset patients with type 1 diabetes [5], and the BABYDIAB, BABYDIET and TeenDiab cohorts of prospectively followed first-degree relatives of patients with type 1 diabetes $[6,7]$. For a pilot study, samples from 32 patients at onset of type 1 diabetes (median age, 12.1 years; interquartile range, 10.2-13.1 years; 31 females), and 32 beta cell autoantibody negative first-degree relatives of patients with type 1 diabetes (median age, 11.9 years; interquartile range, 10.4-13.1 years; 31 females) were used. For an extended study, samples from 1,078 participants were measured. These included 269 beta cell autoantibody positive patients at onset of type 1 diabetes (median age, 10.4 years; interquartile range, 7.1-13.4 years; 124 females), 94 beta cell autoantibody negative patients at onset of type 1 diabetes (median age, 12.4 years; interquartile range, 8.7-14.6 years; 42 females), 503 beta cell autoantibody negative first-degree relatives of patients with type 1 diabetes (median age, 3.2 years; interquartile range, 2.1-5 years; 249 females), 98 relatives with single antibodies to insulin, GAD65, insulinoma antigen-2 (IA-2) or zinc transporter 8 (ZnT8) (median age, 11.7 years; interquartile range, 6.4-14.1 years; 61 females), and 114 relatives with multiple beta cell autoantibodies (median age, 7.4 years; interquartile range, 4.2-11.2 years; 48 females). Of the relatives, 78 had developed diabetes on follow-up. The ethical committees of Bavaria or the LudwigMaximilians University approved the studies, which were carried out in accordance with the Declaration of Helsinki, as revised in 2000. Informed, written consent was obtained from patients or parents of participants.

Cloning and expression of tetraspanin 7 protein Full length tetraspanin 7 and extracellular domain (ED) fragments corresponding to amino acids 41 to 56 (ED1) and 113 to 213 (ED2) were cloned from human islet cDNA. The fragments were $\mathrm{NH}_{2}$-terminally fused to NanoLuc. NanoLuc-tetraspanin 7 full length and untagged tetraspanin 7 full length were cloned into the expression vector pCMV6-AC-IRES-GFP-Puro (Origene, Rockville, MD, USA); and NanoLuc-ED1 and NanoLuc-ED2 were cloned into the pTNT Vector (Promega, Mannheim, Germany). ED1 and ED2 were expressed by in vitro transcription/translation (Promega). Full length NanoLuctetraspanin 7 and untagged full length tetraspanin 7 were expressed in HEK 293T cells by transfecting $2 \mu \mathrm{g}$ expression vector DNA using polyethylenimine (Polysciences, Warrington, PA, USA). Cell pellets were lysed in $1 \%$ Triton $\mathrm{X} 100$ lysis buffer $\left(100 \mu \mathrm{l} / 10^{6}\right.$ cells $)$ for $30 \mathrm{~min}$ at $4^{\circ} \mathrm{C}$. After centrifugation (10 min at $16,000 \mathrm{~g}$ ), the supernatant was collected and used in immunoassays.

Luciferase immunoprecipitation assay for tetraspanin 7 autoantibodies Serum $(2 \mu \mathrm{l})$ was added to duplicate wells of a 96-well microfiltration plate (Merck Millipore, Darmstadt, Germany) containing $23 \mu \mathrm{l}$ of assay buffer (Tris buffered saline $\mathrm{pH} 7.2$ plus $0.1 \%$ Tween 20 , and additional $0.05 \%$ Triton X100 for full length tetraspanin 7) containing 5 million counts per second of NanoLuc-tagged antigen. Plates were incubated in the dark at room temperature for 2 h. Protein-A Sepharose (GE Healthcare, Freiburg, Germany) equivalent to $1.5 \mathrm{mg}$ per well and pre-swollen in assay buffer plus $0.1 \%$ BSA low IgG (Life Technologies, Darmstadt, Germany) was added, and plates shaken (300 rev/min) at $4^{\circ} \mathrm{C}$ for $1 \mathrm{~h}$. Wells were washed ten times
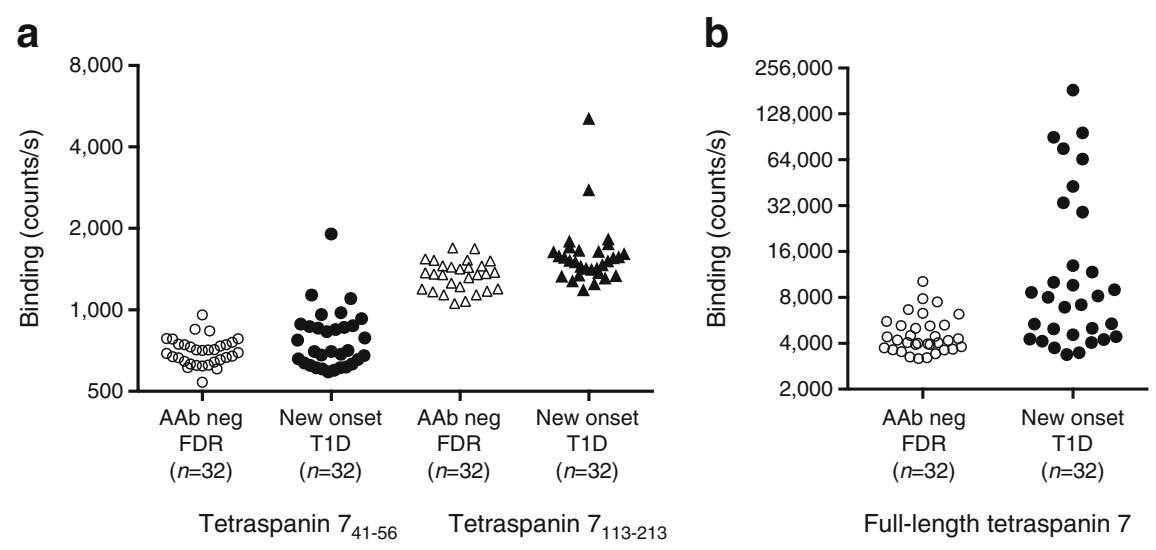

Fig. 1 Autoantibody binding to tetraspanin 7 fragments and full length tetraspanin 7 protein. Light units expressed as counts/s shown on the $y$ axis in luciferase immunoprecipitation assays using (a) NanoLuc-tagged tetraspanin 7 External Domain $41-56$ (ED1; circles), and NanoLuc-tagged tetraspanin 7 External Domain 113-213 (ED2; triangles), both expressed as in vitro transcribed and translated proteins, and (b) NanoLuc-tagged full length tetraspanin 7 expressed in mammalian cells, for sera from beta cell autoantibody negative relatives of patients with type 1 diabetes (AAb neg FDR; open symbols) and patients at onset of type 1 diabetes (New onset T1D; filled symbols) 
with $200 \mu$ l assay buffer. Immunoprecipitated activity was measured after the addition of NanoLuc Glo luciferase substrate (Promega). Binding to the full length tetraspanin 7 was expressed as arbitrary units/ml that were determined from a calibration curve of six calibrators prepared from a pool of positive sera and a pool of negative sera which were included on every plate. For samples with values above 5 units $/ \mathrm{ml}$, the inhibitable binding was measured by performing the assay with (inhibited) and without (uninhibited) lysate from cells that expressed untagged full length tetraspanin 7 . The difference between the immunoprecipitated signals without untagged full length tetraspanin 7 and with excess untagged full length tetraspanin 7 was used to calculate the inhibitable tetraspanin 7 units/ml from a calibration curve. Positivity for tetraspanin 7 autoantibodies was defined as inhibitable binding that identified the upper centile of the 503 beta cell autoantibody negative relatives.

Statistical analysis Comparisons of tetraspanin 7 autoantibody binding between participant groups were performed using the Student's $t$ test. The Kaplan-Meier survival analysis with logrank test was used to compare progression to diabetes between groups. For all tests, a two-tailed $p$ value of 0.05 was considered significant.

\section{Results}

In the pilot study, patients with type 1 diabetes had increased binding to tetraspanin $7 \operatorname{ED} 1(p=0.047), \operatorname{ED} 2(p=0.025)$ and to full length tetraspanin $7(p=0.005)$ as compared with sera from beta cell autoantibody negative relatives (Fig. 1). High signals were only obtained with the full length protein. We, therefore, selected the full length tetraspanin 7 immunoassay

Fig. 2 Autoantibodies to full length tetraspanin 7. (a) Binding to NanoLuc-tagged full length tetraspanin 7 (units/ml) shown on the $y$-axis for beta cell autoantibody negative first-degree relatives of patients with type 1 diabetes (AAb neg FDR), single beta cell autoantibody positive relatives (single AAb pos FDR), multiple beta cell autoantibody positive relatives (multiple AAb pos FDR), beta cell autoantibody negative patients at onset of type 1 diabetes (AAb neg T1D), and beta cell autoantibody positive patients at onset of type 1 diabetes (AAb pos T1D). The dotted horizontal line represents the 99th centile of beta cell autoantibody negative relatives at 15 units $/ \mathrm{ml}$. The dashed line is set at 5 units $/ \mathrm{ml}$ and was used to select samples for measurement of inhibitable tetraspanin 7 antibodies. (b) Inhibitable tetraspanin 7 antibody values measured using a competition assay. The dotted horizontal line represents the combined 99th centile of the 503 beta cell autoantibody negative relatives in the binding and competition assays, defined as 1.4 inhibitable units $/ \mathrm{ml}$. (c) Progression to diabetes shown as the probability of type 1 diabetes ( $y$-axis) during follow-up ( $x$-axis) in multiple beta cell autoantibody positive first-degree relatives with (solid line) and without (dashed line) tetraspanin 7 antibodies $(p=0.38)$. Numbers under the $x$ axis refer to the remaining participants at the time of follow-up in each group for further assessment of autoantibody prevalence. The interassay coefficient of variation for this assay was $12.7 \%$ at 8 units $/ \mathrm{ml}$.

In the extended study, increased binding to full length tetraspanin 7, as compared with binding in beta cell autoantibody negative relatives, was observed in samples from the multiple beta cell autoantibody positive relatives $(p<0.0001)$ and beta cell autoantibody positive patients $(p<0.0001)$, but not from the single beta cell autoantibody positive relatives or the beta cell autoantibody negative patients (Fig. 2a).

The specificity of binding for all samples with $>5$ units $/ \mathrm{ml}$ was assessed by competition (Fig. 2b). Binding was inhibitable in the majority of the tested samples from multiple

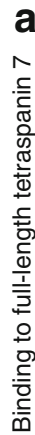
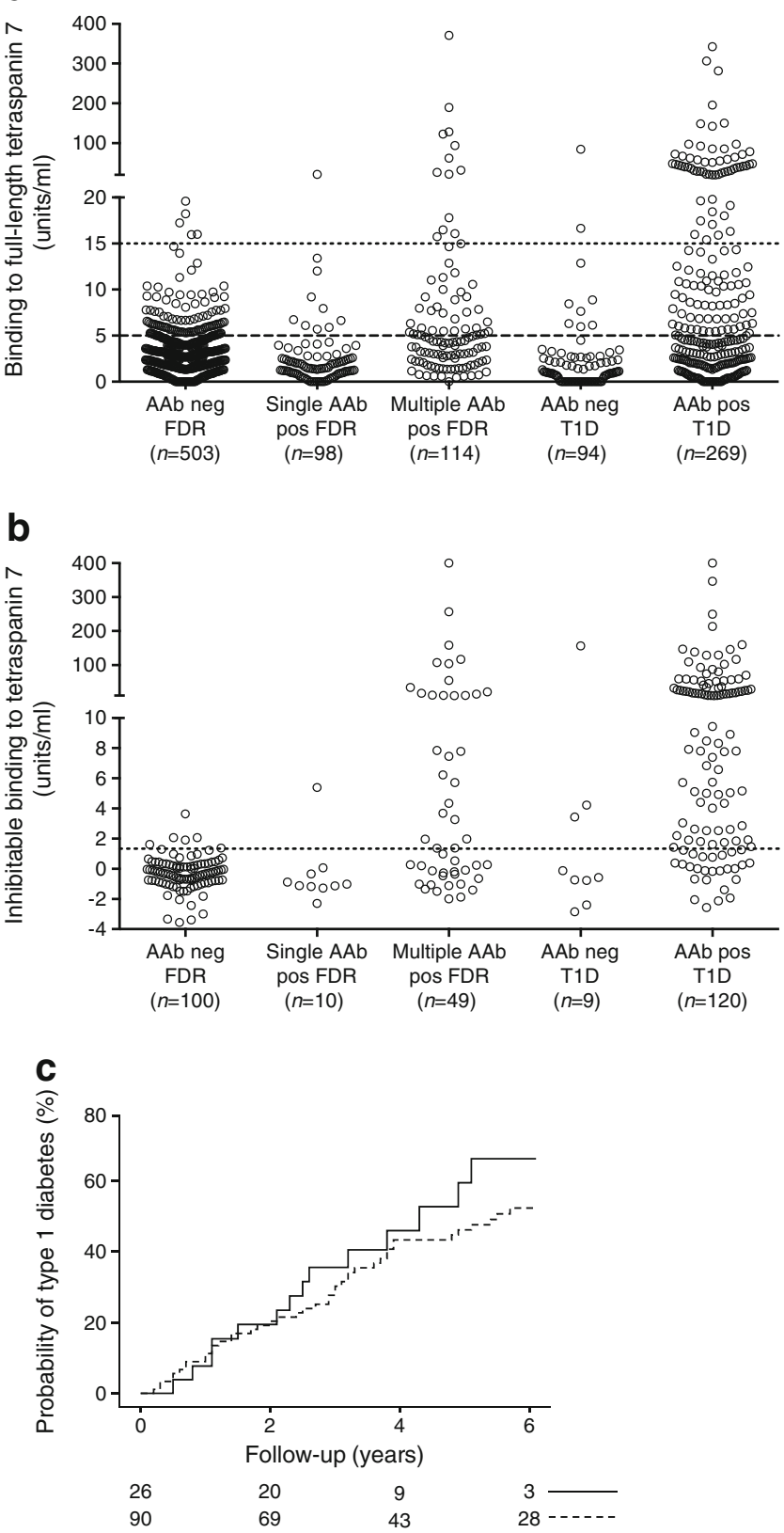
beta cell autoantibody positive relatives and beta cell autoantibody positive patients, but not other groups. Samples that were not inhibited did not bind to NanoLuc or to other NanoLuc fusion proteins (data not shown). Overall, 5 (1\%) of the 503 beta cell autoantibody negative relatives had binding that could be inhibited at a degree that corresponded to $>1.4$ inhibitable binding units $/ \mathrm{ml}$, which was defined as the threshold for positivity. Tetraspanin 7 autoantibodies were observed in $1(1.0 \%)$ of 98 single beta cell autoantibody positive relatives, $25(21.9 \%)$ of 114 multiple beta cell autoantibody positive relatives, $3(3.2 \%)$ of 94 beta cell autoantibody negative patients and 95 (35.3\%) of 269 beta cell autoantibody positive patients. The prevalence of tetraspanin 7 autoantibodies was similar in patients who had GAD autoantibodies (34.4\%), IA-2 autoantibodies (39.8\%) or ZnT8 autoantibodies (38.4\%), and in beta cell autoantibody positive patients with $(38 \%)$ or without $H L A-D R B 1 * 04$ alleles $(32 \%)$. No difference in the progression to clinical diabetes was observed between multiple beta cell autoantibody positive relatives with (66\% within 5 years; $95 \% \mathrm{CI}, 44-88 \%$ ) or without tetraspanin 7 autoantibodies (48\% within 5 years; $95 \%$ CI, 36-60\%; $p=0.38$; Fig. 2c).

\section{Discussion}

Autoantibodies to tetraspanin 7 were found in a minority of patients with type 1 diabetes and beta cell autoantibody positive relatives. Tetraspanin 7 autoantibodies were almost exclusively found in patients with other beta cell autoantibodies, and in relatives who were positive for two or more other beta cell autoantibodies. Although larger studies are required, tetraspanin 7 autoantibody positivity did not appear to stratify risk for clinical diabetes in beta cell autoantibody positive relatives. The higher frequency of tetraspanin 7 autoantibodies in patients at onset of diabetes, as compared with multiple beta cell autoantibody positive children who subsequently developed diabetes, may suggest that tetraspanin 7 autoantibodies appear late in the preclinical phase of type 1 diabetes. However, the cross-sectional measurements performed in our study did not allow us to formally test this. We also did not measure tetraspanin 7 autoantibodies in adult onset diabetes. Therefore, it is possible that tetraspanin 7 autoantibodies may be useful in adult autoimmune diabetes, which often presents with single GAD autoantibodies.

Of potential relevance to pathogenesis, the limited binding studies performed with fragments of tetraspanin 7 suggested that some antibodies bind to epitopes within the external domains of the protein. Such antibodies could directly mediate beta cell death or affect beta cell function, and, therefore, warrant further investigation. Since there are discrepancies between binding to glima 38 and recombinant tetraspanin 7 [4], comparison amongst assays, including assays that use non-tagged protein to avoid binding interference, is also recommended. We conclude that tetraspanin 7 is a relevant autoantigen in the pathogenesis of type 1 diabetes. Nevertheless, although increased sensitivity may be obtained with other methods, the measurement of tetraspanin 7 autoantibodies is currently likely to provide limited additional value to existing beta cell autoantibody measurements for the diagnosis of early type 1 diabetes in childhood.

Acknowledgements The work was supported by the JDRF (No 172012-16), the Helmsley Charitable Trust (2015PG-T1D072) and by grants from the German Federal Ministry of Education and Research (BMBF) to the German Center for Diabetes Research (DZD e.V.). E Bonifacio is supported by the DFG Research Center and Cluster of Excellence Center for Regenerative Therapies Dresden (FZ 111). The tetraspanin 7 antigen is available on request from the corresponding author.

Funding This work was funded by the JDRF (No 17-2012-16) and the Leona M and Harry B Helmsley Charitable Trust (2015PG-T1D072)

Duality of interest The authors declare that there is no duality of interest associated with the manuscript.

Contribution statement EB and AGZ designed the study, contributed to the conduct of the study, the acquisition, analysis and interpretation of data, and drafted, reviewed and approved the manuscript. DW, AE, SJ, $\mathrm{AG}, \mathrm{CWe}, \mathrm{TT}$, and CWi contributed to the acquisition, analysis and interpretation of data, and drafted, reviewed and approved the manuscript. $\mathrm{EB}$ is the guarantor of this work.

\section{References}

1. Aanstoot HK, Kang SM, Kim J et al (1996) Identification and characterization of glima 38, a glycosylated islet cell membrane antigen, which together with GAD65 and IA2 marks the early phases of autoimmune response in type 1 diabetes. J Clin Invest 97:2772-2783

2. Roll U, Turck CW, Gitelman SE et al (2000) Peptide mapping and characterisation of glycation patterns of the glima 38 antigen recognised by autoantibodies in type I diabetic patients. Diabetologia 43:598-608

3. Winnock F, Christie MR, Batstra MR et al (2001) Autoantibodies to a $38-\mathrm{kDa}$ glycosylated islet cell membrane-associated antigen in (pre)type 1 diabetes: association with IA-2 and islet cell autoantibodies. Diabetes Care 24:1181-1186

4. McClaughlin K, Richardson CC, Ravishankar A et al (2016) Identification of tetraspanin-7 as a target of autoantibodies in type 1 diabetes. Diabetes. doi:10.2337/db15-1058

5. Thümer L, Adler K, Bonifacio E et al (2010) German new onset diabetes in the young incident cohort study: DiMelli study design and first-year results. Rev Diabet Stud 7:202-208

6. Ziegler AG, Bonifacio E, Babydiab-Babydiet Study Group (2012) Age-related islet autoantibody incidence in offspring of patients with type 1 diabetes. Diabetologia 55:1937-1993

7. Ziegler AG, Meier-Stiegen F, Winkler C, Bonifacio E, the TEENDIAB Study Group (2012) Prospective evaluation of risk factors for the development of islet autoimmunity and type 1 diabetes during puberty - TEENDIAB: study design. Pediatr Diabetes 13 : $419-424$ 